\title{
Search for doubly-charmed baryons and study of charmed-strange baryon states at Belle
}

\author{
Y. Kato*† \\ KMI, Nagoya University \\ E-mail: kato@hepl.phys.nagoya-u.ac.jp
}

\begin{abstract}
We present the studies of charmed baryons at the Belle experiment. We search for the doubly charmed baryon $\Xi_{c c}^{+}$in the $\Lambda_{c}^{+} K^{-} \pi^{+}$and $\Xi_{c}^{0} \pi^{+}$final states, and do not find any significant signals. We search for $\Xi_{c}(3055)^{+}$and $\Xi_{c}(3123)^{+}$in the $\Lambda_{c}^{+} K^{-} \pi^{+}$final state with intermediate $\Sigma_{c}(2455)^{++}$or $\Sigma_{c}(2520)^{++}$resonances. We observe a significant peak corresponding to the $\Xi_{c}(3055)^{+}$while no signature of the $\Xi_{c}(3123)^{+}$is seen. We observe $\Xi_{c}(3055)^{+/ 0}$ and $\Xi_{c}(3080)^{+/ 0}$ decays into $\Lambda D^{+/ 0}$ for the first time. This is also the first observation of the $\Xi_{c}(3055)^{0}$. We measure the width of the $\Xi_{c}(2645)^{+}$. It is $2.6 \pm 0.2$ (stat) \pm 0.4 (syst) MeV/c ${ }^{2}$, which is the first significant measurement.
\end{abstract}

XXII. International Workshop on Deep-Inelastic Scattering and Related Subjects,

28 April - 2 May 2014

Warsaw, Poland

\footnotetext{
* Speaker.

${ }^{\dagger}$ for the Belle collaboration.
} 


\section{Introduction}

A Charmed baryon is a good laboratory for the understanding of the low energy QCD. Because the mass of the charm quark is significantly larger than its momentum inside the hadron, we can study the static potential between quarks from the doubly charmed baryons. Also, the mass of the charm quark is much heavier than u,d,s quarks and this yields small spin-spin interaction. Therefore, we expect in the singly charmed baryon, light quarks form a di-quark. In recent years, there has been much progress in the spectroscopy of the charmed baryons driven mainly by Belle and BaBar experiments. All the singly charmed baryon ground states predicted by constituent quark model have been discovered and a number of excited states has been also discovered. However, there are still many things to be revealed in this field. There is no established doubly charmed baryon state. There are still many excited states whose experimental evidence is not strong enough to be firmly established.

In this paper, we report the studies of charmed baryons using a data sample with an integrated luminosity of $980 \mathrm{fb}^{-1}$ collected with the Belle detector [1] at the KEKB asymmetric-energy $e^{+} e^{-}$ collider 记].

\section{Search for doubly charmed baryon}

We search for the doubly charmed baryon $\Xi_{c c}^{+}$in the $\Lambda_{c}^{+} K^{-} \pi^{+}$and $\Xi_{c}^{0} \pi^{+}$final states. The $\Lambda_{c}^{+}$ candidate is reconstructed via the decay to $p K^{-} \pi^{+}$and $p K_{S}^{0}$ [3]. $\Xi_{c}^{0}$ candidate is reconstructed via the decay into $\Xi^{-} \pi^{+}, \Lambda K^{-} \pi^{+}$and $p K^{-} K^{-} \pi^{+}$. In order to reduce the combinatorial background, the scaled momentum $x_{p}=p^{*} / \sqrt{s / 4-m^{2}}$, where $p^{*}$ is the CM momentum of a $\Xi_{c}^{*}$ candidate, $s$ is CM energy squared and $m$ is mass of the $\Xi_{c}^{*}$ candidate, is required to be greater than 0.5 for $\Lambda_{c}^{+} K^{-} \pi^{+}$final state and 0.45 for $\Xi_{c}^{0} \pi^{+}$final state.

Figure 1 (a) shows the $M\left(\Lambda_{c}^{+} K^{-} \pi^{+}\right)$distribution with all the selection criteria applied. We evaluate the statistical significance of the $\Xi_{c c}^{+}$from the formula $\sqrt{-2 \ln \left(\mathscr{L}_{0} / \mathscr{L}\right)}$, where $\mathscr{L}_{0}$ is the likelihood for the un-binned extended maximum likelihood fit without the signal component and $\mathscr{L}$ is the likelihood for the fit with the signal component included. The statistical significance is lower than $3 \sigma$ in the mass range of 3.2-4.0 GeV/c ${ }^{2}$. Figure 1 (b) shows the $95 \%$ confidence level (C.L.) upper limit for the product of the cross section and branching fraction to the $\Lambda_{c}^{+} K^{-} \pi^{+}$state for the $\Xi_{c c}^{+}$produced with the $0.5<x_{p}<1.0$ condition,

$$
\sigma_{\mathscr{B}} \equiv \sigma\left(e^{+} e^{-} \rightarrow \Xi_{c c}^{+} X\right) \times \mathscr{B}\left(\Xi_{c c}^{+(+)} \rightarrow \Lambda_{c}^{+} K^{-} \pi^{+}\right)
$$

as a function of the mass. They are 4.1-25.0 fb. Figure 2 (a)-(c) show the $M\left(\Xi_{c}^{0} \pi^{+}\right)$distribution for each decay mode of the $\Xi_{c}^{0}$. We perform simultaneous fit for each $\Xi_{c}^{0}$ decay mode with fixing the relative signal yield determined from $\Xi_{c}(2645)^{+} \rightarrow \Xi_{c}^{0} \pi^{+}$. The statistical significance for the mass around $3.553 \mathrm{GeV} / c^{2}$ is found to be $3.2 \sigma$. Because we scan a wide mass range, we perform a pseudo-experiment test to evaluate the probability of observing a peak with statistical significance higher then $3.2 \sigma$. The probability to observe a peak with a significance higher than $3.2 \sigma$ in one pseudo-experiment is $26 \%$. Therefore, the local statistical significance is $3.2 \sigma$ is not high enough to claim it as an evidence of the $\Xi_{c c}^{+}$. Figure 2 (d) shows the $95 \%$ C.L. upper limit for the product 
of the cross section and branching fractions for the $\Xi_{c c}$ produced with $0.45<x_{p}<1.0$ condition,

$$
\begin{aligned}
\sigma_{\mathscr{B}^{2}} \equiv & \sigma\left(e^{+} e^{-} \rightarrow \Xi_{c c}^{+} X\right) \times \mathscr{B}\left(\Xi_{c c}^{+(+)} \rightarrow \Xi_{c}^{0} \pi^{+}\right) \\
& \times \mathscr{B}\left(\Xi_{c}^{0} \rightarrow \Xi^{-} \pi^{+}\right)
\end{aligned}
$$

They are $0.076-0.35 \mathrm{fb}$ depending on the mass.
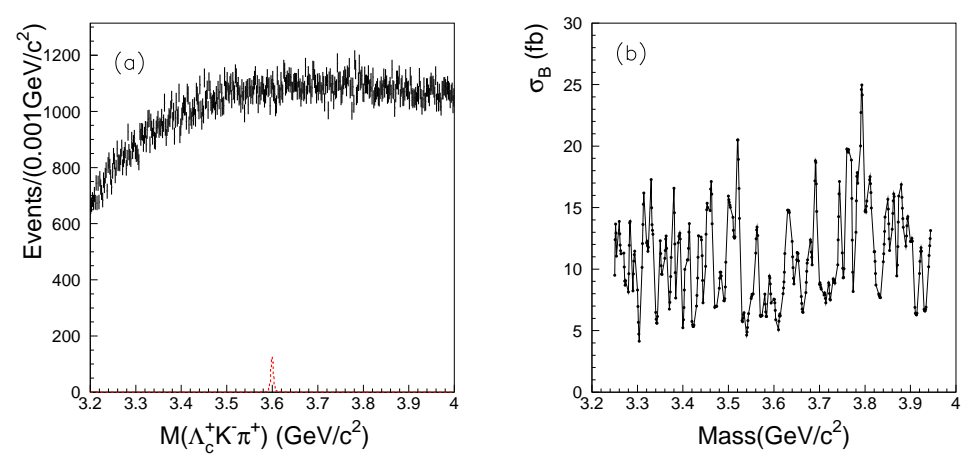

Figure 1: (a) $M\left(\Lambda_{c}^{+} K^{-} \pi^{+}\right)$distribution. Vertical error bars are from data and The dashed histograms are from a MC sample with a mass of $\mathrm{M}\left(\Xi_{c c}^{+}\right) 3.60 \mathrm{GeV} / c^{2}$ and a production cross section $\sigma\left(e^{+} e^{-} \rightarrow \Xi_{c c}^{+(+)} X\right)$ of $500 \mathrm{fb}$ and $\mathscr{B}\left(\Xi_{c c}^{+(+)} \rightarrow \Lambda_{c}^{+} K^{-} \pi^{+}\left(\pi^{+}\right)\right)$of $5 \%$. (b) $95 \%$ C.L. upper limit of $\sigma_{\mathscr{B}}$ as a function of the mass with a $1 \mathrm{MeV} / c^{2}$ step for $\Xi_{c c}^{+}$

\section{Study of the excited $\Xi_{c}$ states in $\Lambda_{c}^{+} K^{-} \pi^{+}$final state}

The Belle collaboration reported evidence of two excited states, $\Xi_{c}(2980)$ and $\Xi_{c}(3080)$, in the $\Lambda_{c}^{+} K^{-} \pi^{+}$and $\Lambda_{c}^{+} K_{S}^{0} \pi^{-}$final states [4]. These states were confirmed by BaBar collaboration later [5]. In the same paper, BaBar also claimed evidence of two resonances, $\Xi_{c}(3055)^{+}$and $\Xi_{c}(3123)^{+}$, through intermediate $\Sigma_{c}(2455)^{++} K^{-}$and $\Sigma_{c}(2520)^{++} K^{-}$final states. The existence of the $\Xi_{c}(3055)^{+}$and $\Xi_{c}(3123)^{+}$are reported only by BaBar collaboration and an independent search by another experiment is necessary. In this study, we require the $x_{p}>0.7$. Figure 1 (a) shows the The $M\left(\Lambda_{c}^{+} K^{-} \pi^{+}\right)$distribution with $\Sigma_{c}(2455)^{++}$selection. We can see clear peak corresponding to the $\Xi_{c}(3055)^{+}$. The statistical significance of the $\Xi_{c}(3055)^{+}$is $6.6 \sigma$. The mass is measured to be $3058.1 \pm 1.0$ (stat) \pm 2.1 (sys) $\mathrm{MeV} / \mathrm{c}^{2}$ and width is measured to be $9.7 \pm 3.4$ (stat) \pm 3.3 (sys) $\mathrm{MeV} / \mathrm{c}^{2}$, which are consistent with previous measurements. Figure 3 (b) shows the The $M\left(\Lambda_{c}^{+} K^{-} \pi^{+}\right)$distribution with $\Sigma_{c}(2520)^{++}$selection. We can not see any peaking structure corresponding to $\Xi_{c}(3123)^{+}$. The $95 \%$ C.L. upper limit for the product of the cross section and branching fraction of $\Lambda_{c}^{+}$produced with $x_{p}>0.7$ condition,

$$
\sigma_{\mathscr{B} \Lambda_{c}^{+}} \equiv \sigma\left(e^{+} e^{-} \rightarrow \Xi_{c}(3123)^{+} X\right) \times \mathscr{B}\left(\Lambda_{c}^{+} \rightarrow p K^{-} \pi^{+}\right)
$$

is obtained to be $0.34 \mathrm{fb}$, which is much smaller than that one quoted in Ref. [5] ( $1.6 \pm 0.6 \pm 0.2$ fb). 

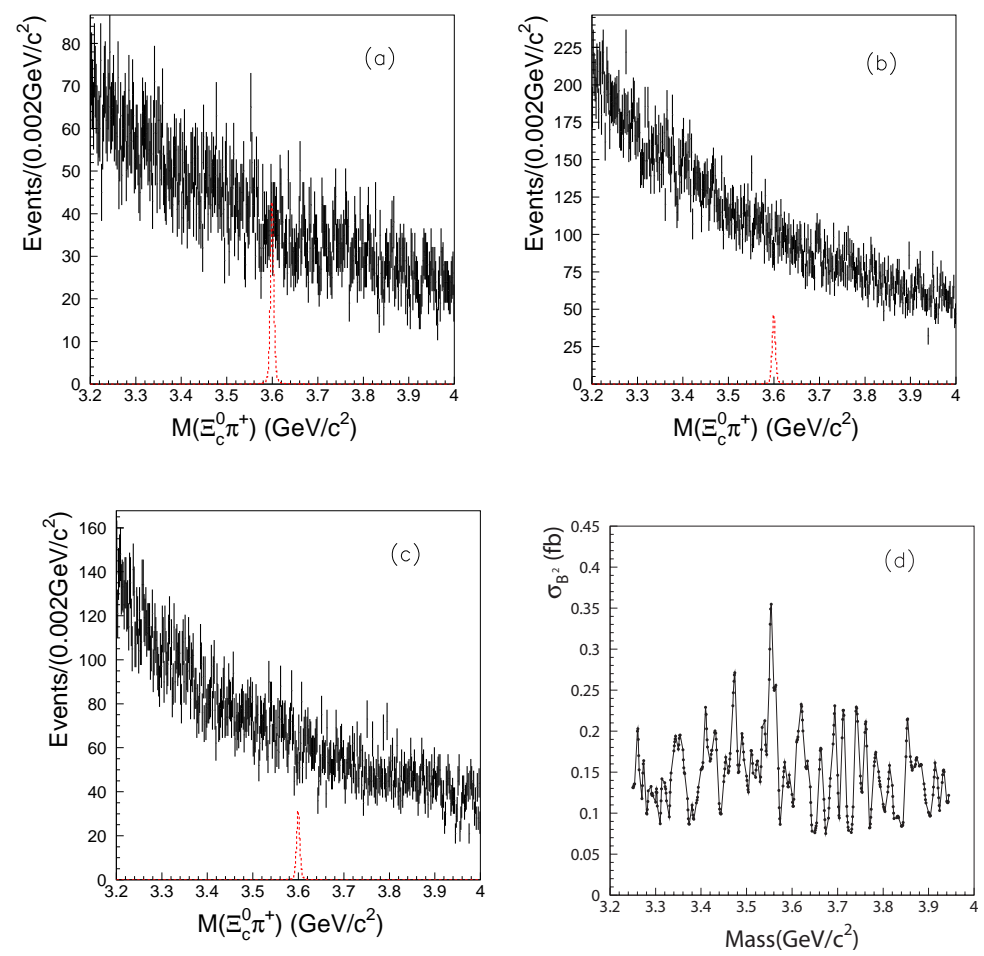

Figure 2: (a)-(c): $M\left(\Xi_{c}^{0} \pi^{+}\right)$distribution in the $\Xi_{c c}^{+}$search region for $\Xi_{c}^{0} \rightarrow$ (a) $\Xi^{-} \pi^{+}$, (b) $\Lambda K^{-} \pi^{+}$, (c) $p K^{-} K^{-} \pi^{+}$. The vertical error bars are from data. The dashed histograms are from a MC sample with a mass of $\mathrm{M}\left(\Xi_{c c}^{+}\right) 3.60 \mathrm{GeV} / c^{2}$ and a production cross section $\sigma\left(e^{+} e^{-} \rightarrow \Xi_{c c}^{+} X\right)$ of $500 \mathrm{fb}$ and $\mathscr{B}\left(\Xi_{c c}^{+} \rightarrow \Lambda_{c}^{+} K^{-} \pi^{+}\left(\pi^{+}\right)\right)$and $\mathscr{B}\left(\Xi_{c}^{0} \rightarrow \Xi^{-} \pi^{+}\right)$of $5 \%$. (d): 95\% C.L. upper limit of the $\sigma_{\mathscr{B}^{2}}$ for $\Xi_{c c}^{+}$as a function of the mass with a $1 \mathrm{MeV} / \mathrm{c}^{2}$ step.

\section{Study of the excited $\Xi_{c}$ states in $\Lambda D^{+/ 0}$ final state}

Currently, all the excited $\Xi_{c}$ are found in the decay mode comprising a charmed baryon and light mesons, for example $\Lambda_{c}^{+} K^{-} \pi^{+}$or $\Xi_{c}^{0} \pi^{+}$. It is very interesting to search for the decay mode comprising a light baryon and a charmed meson. We search for excited $\Xi_{c}$ states in the $\Lambda D^{+/ 0}$ final states. The results of this section are preliminary. $\Lambda$ is reconstructed via the decay into $p \pi^{-}$. $D^{+}$is reconstructed via the decay into $K^{-} \pi^{+} \pi^{+} . D^{0}$ is reconstructed via the decay into $K^{-} \pi^{+}$, $K^{-} \pi^{+} \pi^{+} \pi^{-}$and $K^{-} \pi^{+} \pi^{0}$. Figure 4 shows the $M\left(\Lambda D^{+}\right)$and $M\left(\Lambda D^{0}\right)$ distributions. We can see peaks corresponding to $\Xi_{c}(3055)^{+/ 0}$ and $\Xi_{c}(3080)^{+/ 0}$. The statistical significances are obtained to be $11.9(4.7) \sigma$ for $\Xi_{c}(3055)^{+}\left(\Xi_{c}(3080)^{+}\right)$and $7.6(2.6) \sigma$ for the $\Xi_{c}(3055)^{0}\left(\Xi_{c}(3080)^{0}\right)$. This is the first observation of the $\Xi_{c}(3055)^{0}$. The mass of the $\Xi_{c}(3055)^{0}$ is obtained to be $3059.7 \pm 0.6$ (stat) \pm 0.5 (syst) $\mathrm{MeV} / \mathrm{c}^{2}$ and width is $7.4 \pm 1.9$ (stat) \pm 3.4 (sys) $\mathrm{MeV} / \mathrm{c}^{2}$.

\section{Measurement of the width of $\Xi_{c}(2645)^{+}$}

Currently, no significant measurement for the width of $\Xi_{c}(2645)^{+}$exists. Only the upper limit of $3.1 \mathrm{MeV} / c^{2}$ is given [6]. Figure 5 shows the $M\left(\Xi_{c}^{0} \pi^{+}\right)$distributions for each decay mode of $\Xi_{c}^{0}$. 

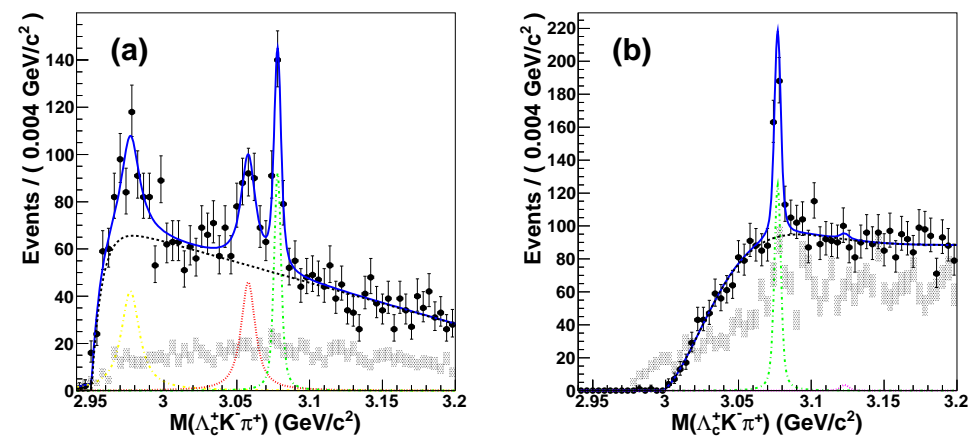

Figure 3: (a) The $M\left(\Lambda_{c}^{+} K^{-} \pi^{+}\right)$distribution with $\Sigma_{c}(2455)^{++}$selection. The dots with error bars show the distribution for the $\Sigma_{c}(2455)^{++}$selected whereas the rectangles show the distribution for the $\Sigma_{c}(2455)^{++}$ sideband region. Blue line shows the fit result. Black, yellow, red, and green lines show the contributions from the background, $\Xi_{c}(2980)^{+}, \Xi_{c}(3055)^{+}$, and $\Xi_{c}(3080)^{+}$, respectively. (b) The $M\left(\Lambda_{c}^{+} K^{-} \pi^{+}\right)$distribution with $\Sigma_{c}(2520)^{++}$selection. The dots with error bars show the distribution for $\Sigma_{c}(2520)^{++}$selected region whereas the rectangles show the distribution for the $\Sigma_{c}(2520)^{++}$sideband region. Blue line shows the fit result. Black, green, and purple lines show the contributions from the background, $\Xi_{c}(3080)^{+}$, and $\Xi_{c}(3123)^{+}$, respectively.
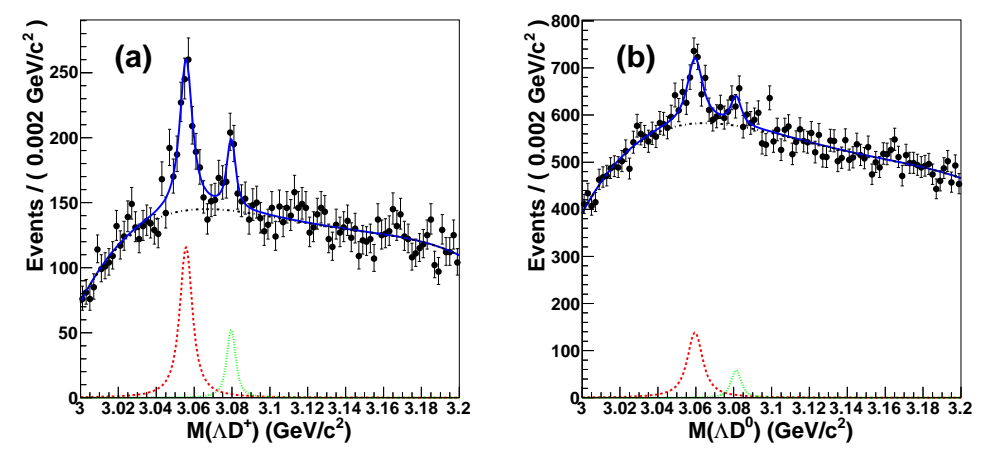

Figure 4: (a): $M\left(D^{+} \Lambda\right)$ distribution. (b): $M\left(D^{0} \Lambda\right)$ distribution. Blue line shows the fitting result. Black, red, and green lines show the background, $\Xi_{c}(3055)^{+/ 0}$, and $\Xi_{c}(3080)^{+/ 0}$ components, respectively.

We can see clear peaks corresponding to $\Xi_{c}(2645)^{+}$. The bump around $2.68 \mathrm{GeV} / c^{2}$ originates from the process $\Xi_{c}(2790)^{+} \rightarrow \Xi_{c}^{\prime 0} \pi^{+} \rightarrow \Xi_{c}^{0} \gamma \pi^{+}$with the $\gamma$ missing in the reconstruction. We perform simultaneous un-binned extended maximum likelihood fit with fixing mass and width of the $\Xi_{c}(2645)^{+}$. The width of the $\Xi_{c}(2645)^{+}$is obtained to be $2.6 \pm 0.2$ (stat) \pm 0.4 (sys) MeV/c ${ }^{2}$.

\section{Summary}

We report the study of charmed baryons at the Belle experiment. We do not find doubly charmed baryon $\Xi_{c c}^{+}$in the $\Lambda_{c}^{+} K^{-} \pi^{+} \Xi_{c}^{0} \pi^{+}$decay modes and put stringent upper limit on the product of the cross section and branching fractions. We confirm the existence of the $\Xi_{c}(3055)^{+}$ while we do not find significant signal of the $\Xi_{c}(3123)^{+}$which was previously reported by BaBar 

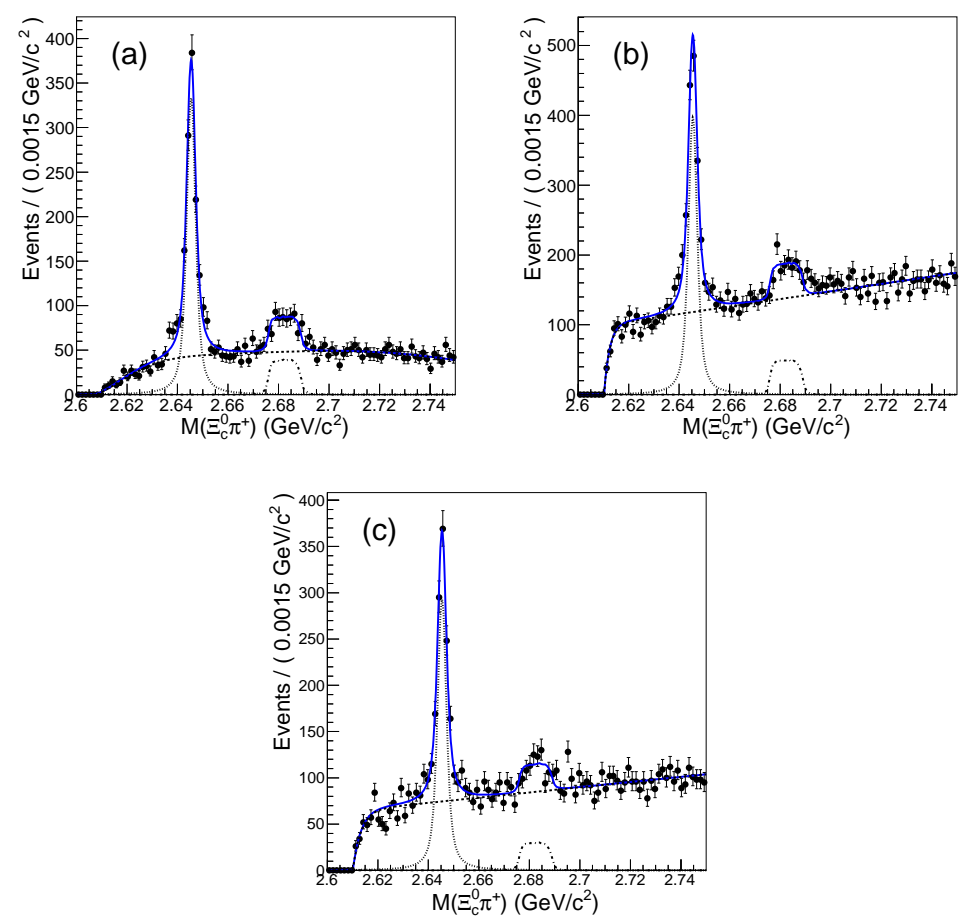

Figure 5: $M\left(\Xi_{c}^{0} \pi^{+}\right)$distributions below the $\Xi_{c c}$ search region for (a) $\Xi_{c}^{0} \rightarrow \Xi^{-} \pi^{+}$, (b) $\Xi_{c}^{0} \rightarrow \Lambda K^{-} \pi^{+}$, (c) $\Xi_{c}^{0} \rightarrow p K^{-} K^{-} \pi^{+}$. The solid lines show the fit result. The dashed, dotted, and dash-dotted lines show the contributions from background, $\Xi_{c}(2645)^{+}$, and $\Xi_{c}(2790)^{+}$, respectively.

in the $\Lambda_{c}^{+} K^{-} \pi^{+}$final state. We find the decay of $\Xi_{c}(3055)^{+/ 0}$ and $\Xi_{c}(3080)^{+/ 0}$ into the $\Lambda D^{+/ 0}$ final state. Especially, this is the first observation of the $\Xi_{c}(3055)^{0}$. We perform the first significant measurement for the width of the $\Xi_{c}(2645)^{+}$.

\section{References}

[1] A. Abashian et al. (Belle Collab.), Nucl. Instr. and Meth. A 479, 117 (2002); also see detector section in J.Brodzicka et al., Prog. Theory. Exp. Phys. (2012) 04D001.

[2] S. Kurokawa and E. Kikutani, Nucl. Instr. and. Meth. A499, 1 (2003), and other papers included in this volume; T.Abe et al., Prog. Theor. Exp. Phys. (2013) 03A001 and following articles up to 03A011.

[3] Throughout this paper, the inclusion of the charge-conjugate decay mode is implied unless otherwise stated.

[4] R. Chistov et al. [Belle Collaboration], Phys. Rev. Lett. 97, 162001 (2006) [hep-ex/0606051].

[5] B. Aubert et al. [BaBar Collaboration], Phys. Rev. D 77, 012002 (2008) [arXiv:0710.5763 [hep-ex]].

[6] L. Gibbons et al. [CLEO Collaboration], Phys. Rev. Lett. 77, 810 (1996). 